\title{
Las estrategias de las principales empresas agroindustriales de soja en Argentina 1980-2011: los casos de Bunge, Cargill y LDC desde un enfoque internacional
}

\author{
The strategies of the main soybean agro-industrial companies in Argentina 1980-2011: the cases of Bunge, Cargill \\ and Dreyfus from an international perspective
}

\author{
Federico Daniel Naspleda \\ CONICET, Instituto de Investigaciones en Humanidades y Ciencias Sociales \\ de la Universidad de La Plata (IdHICS). Laboratorio en Estudios en \\ Sociología y Economia del Trabajo (LESET), Argentina \\ fdnaspleda@gmail.com \\ (iD https://orcid.org/0000-0001-9720-4576
}

\section{ReSUMEN:}

En Argentina entre los años 1980-2011 las producciones de aceite y harina de soja crecieron a tal punto de convertirse en las más importantes del mundo. Bunge, Cargill y Dreyfus van terminar liderando la competencia en este rubro por las tácticas desarrolladas en los tres países más productivos: Argentina, Brasil y EE.UU. El objetivo de este trabajo es comprender las estrategias realizadas por estas corporaciones en los tres países de referencia para explicar la formación de una posición competitiva única en Argentina basada en la integración vertical. Se aplican métodos cuantitativos y cualitativos mediante un análisis teórico y descriptivo del contexto mundial y local.

Palabras Clave: Estrategias, Empresas multinacionales, Producción de harina y aceite de soja, Argentina.

\section{Abstract:}

In Argentina, from the eighties to 2011, soybean oil and flour productions grew to the point of becoming the most important in the world. Bunge, Cargill and Dreyfus will end up leading the competition for the tactics developed in the three most productive countries: Argentina, Brazil and the US. The objective of this work is to understand the strategies carried out by these corporations in the three reference countries to explain the formation of a unique competitive position in Argentina. Quantitative and qualitative methods are applied through a theoretical and descriptive analysis of the global and local context.

KEYWORDS: Multinational enterprises, Strategies, Soybean meal and oil production, Argentina.

\section{INTRODUCCIÓN}

En Argentina, la producción de los derivados industriales de soja presentó un crecimiento constante y de largo plazo entre 1980-2011, periodo en el cual la misma se convirtió en una de las tres mayores del mundo.

El objetivo de este trabajo es comprender las estrategias de las principales empresas multinacionales productoras y comercializadoras de aceite y harina de soja entre los años 1980-2011 en Argentina. Se trata de Bunge, Cargill y Dreyfus -a partir de ahora a las tres empresas se las denominará BCD-.

Las acciones de estas multinacionales serán examinadas según un punto de vista global en el cual se toma como referencia la actividad en los tres principales países productores: Argentina, EE. UU. y Brasil. Por lo que este análisis comprende tendencias económicas y sociales que explican importantes transformaciones en el sector en estudio.

La principal hipótesis de este trabajo es la fuerte relación y articulación existente de las tácticas de BCD en los países analizados. Estas estrategias suponen acciones destinadas al control de la posición competitiva de la cadena agroindustrial global mediante la integración vertical productiva y comercial, que se sucedió 
de manera progresiva en los años de estudio. Esto les permitió competir de mejor manera frente a empresas locales que dependían solamente de su producción en el país de estudio, y no contaban con inversiones a lo largo de la cadena de valor agroindustrial.

Por su importancia en Argentina y en el mundo, el sector agroindustrial de derivados de soja fue objeto de importantes estudios de investigación. Los mismos se centraron en primer lugar en los procesos de concentración e internacionalización de estas empresas en Argentina por el aumento de la demanda de sus productos a nivel global (Gutman \& Gorenstein, 2005; Pierri, 2008; Regunaga, 2010; Vidosa, 2020), y/ o como parte de un proceso mundial de búsqueda de reducciones en los costos productivos y logísticos (Gutman \& Gorenstein, 2005; Regunaga, 2010). En segundo lugar, existen otros estudios que investigaron el vínculo existente entre el liderazgo económico del sector agroindustrial y la forma particular que adquirió el crecimiento económico en la Argentina neoliberal, según un proceso de liberalización y extranjerización económica (Dougnac, 2004; Giarracca \& Teubal, 2008; Gorenstein \& Ortiz, 2016; Gras, 2012; Grondona \& Burgos, 2015; López et al., 2010; Regunaga, 2010; Sly, 2017; Vidosa, 2020), y ventajas tecnológicas (Delvenne et al., 2013). Otros autores relacionan estas tendencias en Argentina respecto a América Latina por la formación de un perfil productivo de commodities industriales de carácter regional (Katz \& Stumpo, 2001; Mortimore \& Peres Núñez, 2001), y especializado en alimentos (Gorenstein \& Ortiz, 2016; Gutman \& Gorenstein, 2005; Pierri \& Junior, 2017; Saravia-Matus et al., 2018). En tercer lugar, varios estudios tienen como objeto comprender las transformaciones tecnológicas y organizacionales en Argentina que implicaron el desplazamiento de la producción vertical por la organización en forma de red (Anlló et al., 2015; Bisang, 2011; Bisang et al., 2009). En cuarto lugar, una parte importante de estudios se han centrado en las distintas actividades de las empresas transnacionales orientadas a aumentar la concentración productiva y comercial en el mercado mundial dados los límites que presenta el mismo (Green \& Hervé, 2006; Murphy et al., 2012; Oliver \& Sánchez, 2016; Salerno, 2017; Serra et al., 2010; Wesz Jr, 2016). Estas actividades incluyen actividades financieras (Clapp, 2014; Salerno, 2017), productivas y de infraestructura.

El presente artículo complementa los estudios del tema en un área de vacancia, comprendiendo las estrategias de $\mathrm{BCD}$ en relación al contexto competitivo local y en el de los principales países productores del mundo. Busca explicar las acciones empleadas por estas transnacionales para construir una posición competitiva en el mundo y de esta forma aventajar e incluso desplazar a empresas locales.

Otros aportes del presente trabajo refieren al recorte y conceptualización histórica sobre el que se analizan las estrategias empresarias en Argentina (1980-2011). En un primer momento, durante la década del ochenta, en Argentina se consolidó un proceso de reestructuración productiva en el conjunto de su economía, en el cual los productos agroindustriales de soja crecieron a tal punto de convertirse en uno de los principales productos exportados, en un contexto de crisis de la industrialización por sustitución de importaciones. En un segundo momento, durante la década del noventa y pleno funcionamiento del neoliberalismo (1991-2001), ingresó Dreyfus al país mediante un proceso de inversiones estratégicas que fueron de las más importantes a nivel mundial. En los años siguientes a la crisis del neoliberalismo se adoptaron políticas económicas de tipo de cambio alto y expansión de la demanda -etapa conocida como posneoliberalismo (2002-2011)-, y se profundizaron las inversiones de las BCD. El sector agroindustrial se convirtió en uno de los motores del crecimiento económico argentino, y contó con un nivel tecnológico y competitivo de los más avanzados a nivel mundial. Entonces, el periodo analizado es muy importante para comprender el proceso de ingreso y afianzamiento de las BCD para dominar el mercado mundial, y su gran impacto en la estructura económica del país de estudio.

A continuación, en el primer apartado, se realiza un análisis en el que se identifican las principales tendencias de la producción y competitividad de harinas y aceites en Argentina, Brasil y EE.UU. respecto al mundo. Se comprenden los problemas competitivos que atravesaron las principales empresas en el marco de la internacionalización de la producción, y las principales estrategias adoptadas como salida a su crisis. Se analizan los procesos de inversión de las mismas. 
En el segundo apartado se estudian las estrategias de las BCD y las empresas locales más concentradas en Argentina siguiendo sus inversiones, la concentración del mercado, su producción, el nivel tecnológico, sus exportaciones, entre otros elementos. Se establecen relaciones respecto a las estrategias examinadas en el apartado 2. A su vez, se comprende la evolución de las empresas y del sector agroindustrial sojero respecto a la inserción industrial argentina en el marco de la IP desde los años ochenta. También se analiza la evolución de las BCD respecto a importantes elementos del neoliberalismo, como la competencia coercitiva. Por último, se examina el desempeño de las corporaciones en los años de posneoliberalismo.

\subsection{La PRODUCCIÓN DE ACEITE Y HARINA DE SOJA A NIVEL INTERNACIONAL. ASPECTOS GENERALES DE SU EVOLUCIÓN}

En las últimas décadas se puede observar en el complejo de soja un constante crecimiento en la preparación de productos más elaborados, tendencia que se consolidó ya en el año 2011 en el cual el 85\% de la cosecha mundial de soja fue procesada y convertida en harina de soja, y el $15 \%$ restante fue convertida en aceite de soja (Murphy et al., 2012).

\section{FIGURA 1}

Participación mundial de las exportaciones de aceite de soja, Argentina,

Brasil y EE.UU.; y exportaciones totales en el mundo, en porcentaje,

y producción mundial de aceite de soja (eje derecho en t.). 1988-2011

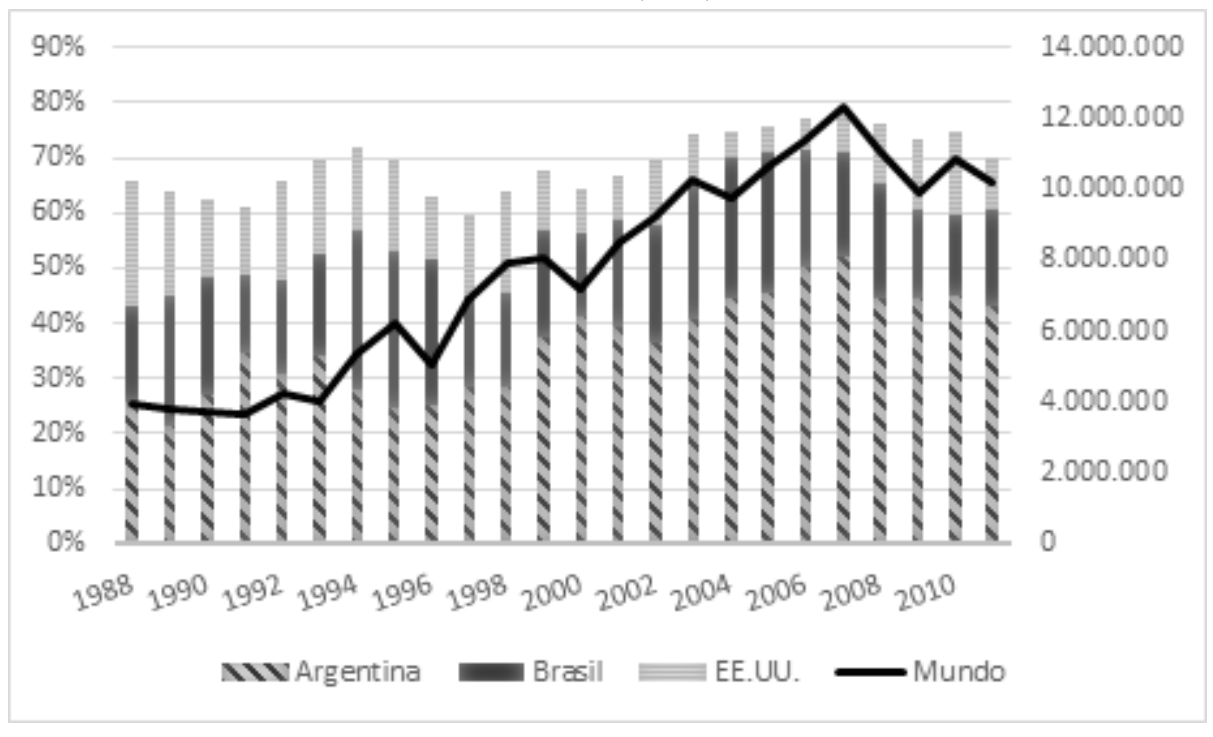

Fuente: elaboración propia a partir de FAO 
FIGURA 2

Participación mundial de las exportaciones de harina de soja en Argentina, Brasil, EE.UU. y el mundo, en porcentaje, y producción mundial de harina (en t.). 1988-2011

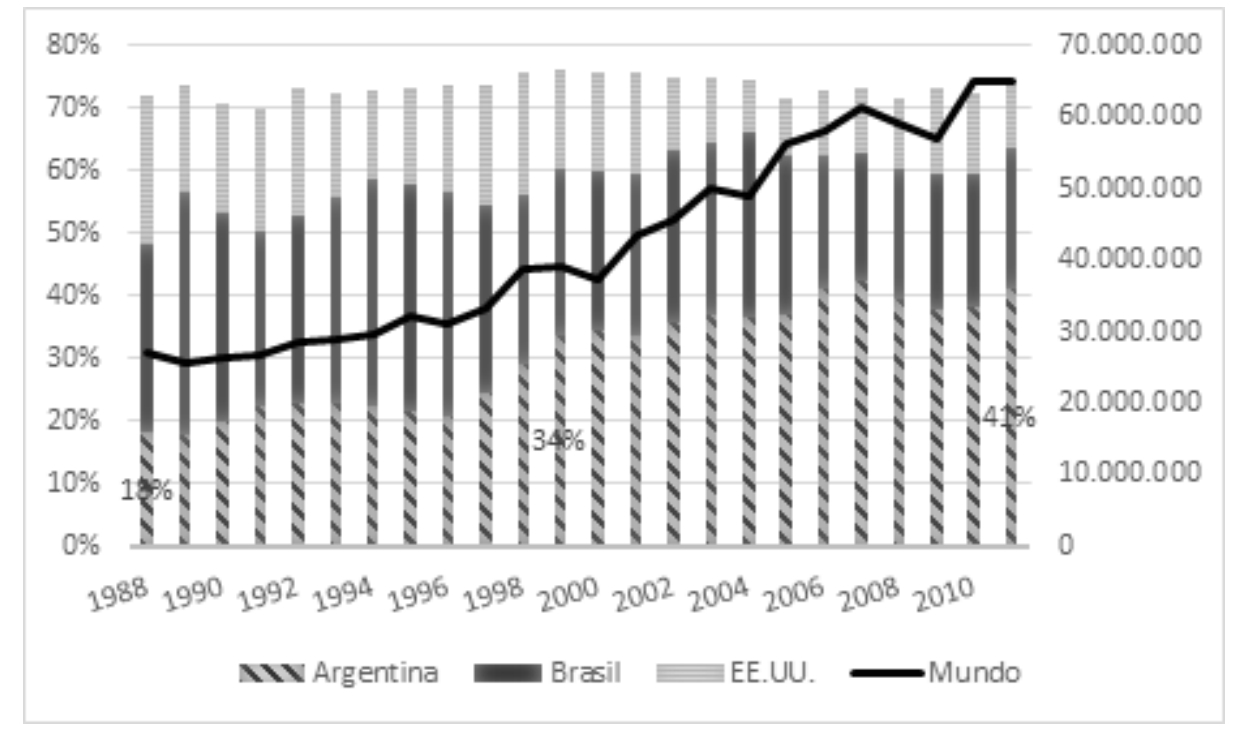

Fuente: elaboración propia en base a FAO

En términos globales, la producción para la exportación de aceite y harina de soja muestra una tendencia de continuo crecimiento en el periodo $1988-2011$ de un $160 \%$ y $141 \%$ respectivamente -figura 1 y 2-. La producción se realiza principalmente en 3 países: Argentina, Brasil y EE.UU. La elaboración de ambos productos en argentina muestra una mayor tendencia a la concentración mundial, aumentando su participación en la producción global desde 1988 hasta 2011 de manera continua; en el caso del aceite la participación mundial pasó del $26 \%$ al 43\%, mientras que las harinas pasaron del 18\% al $41 \%$. El crecimiento en la participación de la producción global de harina de soja en Argentina se corresponde con el decrecimiento de la producción en Brasil y EE.UU., y respecto del aceite de soja por el decrecimiento en EE.UU. y el estancamiento en Brasil.

Es de destacar que en este periodo Brasil y EE.UU. van a liderar la exportación de porotos de soja, mientras que la participación de Argentina en las exportaciones totales de dicho producto va a oscilar en un 25\% (Grondona \& Burgos, 2015).

Por otra parte, la cadena de valor de soja es una red compleja y requiere muchos servicios de apoyo (por ejemplo, infraestructura y procesamiento), lo que crea una gran cantidad de valor económico (Garrett et al., 2013). A su vez requiere una enorme distancia de transporte para satisfacer la demanda global (He et al., 2019).

Estos elementos junto a otros definen diferencias en la competitividad entre los países, y serán descriptos a continuación.

En primer lugar, la dotación de recursos naturales y climas favorables en Argentina, Brasil y EE. UU los convierte en productores de soja a bajo costo (Schnepf et al., 2001).

En segundo lugar, la logística es un elemento clave. En Brasil y Argentina las empresas han centrado más esfuerzos en la expansión de la tierra en lugar de la infraestructura, por esto el principal transporte interno en estos países es por carretera y no por trenes y ríos siendo más costoso. Mientras que en EE.UU. el coste de transporte ha sido más barato por las menores distancias que tienen que realizar los camiones (Informa Economics, 2016). En Argentina el problema de transporte se ha reducido porque la mayor parte de la harina y aceite de soja se produjo cerca de los puertos (Regunaga, 2010). A su vez, la mayoría de la soja de EE.UU. se ha vendido a elevadores y procesadores de soja porque estos dos mercados están bien establecidos y ubicados en áreas donde se cultiva la mayor parte de la cosecha de soja (Council US Soybean Export, 2011). 
En tercer lugar, en Brasil, la tasa impositiva sobre los productos de soja ha sido bastante baja, y las exportaciones de aceite y harina en los últimos años tuvieron una tasa impositiva cero (Garrett et al., 2013). Mientras que en Argentina el esquema de derechos a las exportaciones adoptado por el estado argentino en 2002, 2007 y 2008 fue el más significativo del periodo. El mismo en el último año mencionado pasó a ser para granos del 35\%, y para aceites y harinas del 32\% (Giancola et al., 2009).

En cuarto lugar, la tierra y la mano de obra son más baratos para los productores de América del Sur que para los estadounidenses, lo que también mantiene bajos los costos de producción (Informa Economics, 2016).

En quinto lugar, respecto al tipo de cambio, Argentina y Brasil han depreciado sus monedas después del año 2001, lo que supone una situación más competitiva respecto a EE.UU. (Council US Soybean Export, 2011). En este sentido, los procesadores de soja y los elevadores de exportaciones en Argentina y Brasil compran su soja en moneda local y venden sus productos en dólares estadounidenses siendo menores los costos de producción.

En sexto lugar, los costos operativos desde una dimensión global son un factor estratégico debido a que la rentabilidad del comercio no sólo dependía del precio internacional, sino también de factores como las tasas de transporte, las tasas de cambio de divisa, la propiedad de los elevadores y de otras infraestructuras, así como las políticas públicas respecto a los créditos a la exportación, la ayuda al desarrollo, los subsidios y las políticas fiscales (Green \& Hervé, 2006; Oliver \& Sánchez, 2016).

Estos factores estratégicos muestran la complejidad de la actividad, por lo que muy pocas empresas van a poder diseñar tácticas aprovechando el conjunto de los mismos y destacarse a nivel competitivo.

\subsection{Las estrategias de BCD en Argentina, Brasil y EE.UU.}

Las principales corporaciones agroindustriales de soja, ADM, Bunge, Cargill y Dreyfus, son empresas de larga trayectoria en la comercialización de granos a nivel mundial, que participaron en varias actividades agropecuarias. Desde principio de la década del setenta se vieron obligadas a expandirse hacia una producción más elaborada por la baja en las ganancias en el comercio de granos, el aumento creciente de su demanda de productos agropecuarios y agroindustriales elaborados a nivel mundial, y la creciente rivalidad para producir en vastas regiones del mundo (Baines, 2014; Green \& Hervé, 2006; Murphy et al., 2012).

A nivel global las actividades manufactureras y comerciales sufrieron un cambio inusitado producto del avance de la internacionalización de la producción desde principios de los años setentas, transformación que se acrecentó a lo largo de la etapa en estudio. La misma fue resultado de la expansión de empresas hacia los llamados países "no desarrollados", como intento de solucionar la caída de sus tasas de ganancias en los países centrales (Brenner, 2006). Este fenómeno en el mundo no se dio de manera homogénea, sino que, por un lado, se formaron regiones que presentaron una fuerte dinámica comercial y productiva, como son los casos de los principales países manufactureros del Sudeste y Este asiático dentro de los cuales destaca China (Felipe \& Mehta, 2016). Mientras que por otro lado, América Latina evidenció un claro rezago productivo al perder competitividad frente a los países más dinámicos, con las excepciones de algunas áreas productivas relacionadas a commodities (Mortimore y Peres Núñez, 2001). Este proceso a su vez intensificó la competencia entre las empresas en el mundo. 
TABLA 1

Principales adquisiciones, joint venture, greenfield de Bunge,

Cargill y Dreyfus en Argentina, Brasil y EE.UU. 1980-2011

\begin{tabular}{|c|c|c|c|}
\hline & Bunge & Cargill & Dreyfus \\
\hline 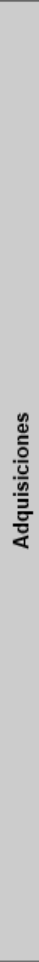 & $\begin{array}{l}1980 \text { Indo, Argentina Cereales y } \\
\text { oleaginosas } \\
1980 \text { tres refinerías de aceite en } \\
\text { EEE.UU. (Bradley, Illinois; } \\
\text { Chattanooga Tennessee; Fort } \\
\text { Worth, Texas) ingresando al } \\
\text { negocio de aceites. } \\
\text {-1997 CEVAL, el mayor productor } \\
\text { de soja de Brasil y empresa lider en } \\
\text { Argentina.1997 IAP, Brasil } \\
\text { (fertilizantes) } \\
\text { 1998 Santista, Brasil (alimentos, } \\
\text { harinas) } \\
\text { 2001 Central Soya (EE.UU.). Una } \\
\text { de las } 5 \text { mayores trituradoras de } \\
\text { soja } \\
\text { 2002 La Plata Cereal S.A., } \\
\text { Argentina. El mayor exportador } \\
\text { mundial de aceite de soja y } \\
\text { alimentos de soja en Argentina } \\
\text { 2002 Manah, Brasil (fertilizantes) } \\
\text {-2002 Oro verde, Brasil } \\
\text { (fertilizantes). } \\
\text { 2002 Fosfertil, Brasil (fertilizantes) } \\
\text {-2002 Cereol (Europa). Tiene } \\
\text { plantas de procesamiento de } \\
\text { semillas oleaginosas en toda } \\
\text { Europa del Este. Esta compra llevó } \\
\text { a Bunge a ser la segunda empresa } \\
\text { de procesamiento de semillas } \\
\text { oleaginosas más grande del mundo } \\
\text { 2007 Santa Juliana, Brasil (caña } \\
\text { de Azúcar) } \\
\text { - Corn Products, EE.UU. (maíz) } \\
\text {-2008 Tate\& Lyle's sugar-trading } \\
\text { arm, fábrica de azúcar y etanol en } \\
\text { Brasil _2009 PASA, Argentina } \\
\text { (fertilizantes) } 2010 \text { Grupo } \\
\text { Moema, Brasil (azúcar y bioenergía) } \\
5 \text { plantas. }\end{array}$ & $\begin{array}{l}\text { 1985, Cargill finalizó la compra de } \\
\text { seis plantas de procesamiento de } \\
\text { soja a Ralston Purina. Se convirtió en } \\
\text { la trituradora de soja más grande de } \\
\text { Estados Unidos. } \\
\text {-1985 Gardinier (fertilizantes) } \\
\text {-1986 Northeast Petroleum } \\
\text { gran5 } 19 \text { Bunge elevadores para } \\
\text { y Minnesota; y una exporado, Dakota } \\
\text { granos de Portland. } \\
\text { 1997 Akzo Salt, Inc., lo que } \\
\text { convierte a Cargill en una de las } \\
\text { empresas de producción y } \\
\text { comercialización de sal más grandes } \\
\text { del mundo } \\
\text {-1998 Monsanto se alió con Cargill y } \\
\text { compró Dekalb También la semillera } \\
\text { Delta \& biotecnología. } \\
\text { 1994 InterMountain Canola, EE.UU. } \\
\text { (aceite) } \\
\text {-1998 Continental, EE.UU. La } \\
\text { transacción incluye las operaciones } \\
\text { de almacenamiento, transporte, } \\
\text { exportación y comercialización de } \\
\text { cereales del Continental en América } \\
\text { del Norte, Europa, América Latina y } \\
\text { Asia " } \\
\text { 2002 Cerestar (cereals) } \\
\text { - 2006 Cerestar (alimentos) } \\
\text { 2008_ Tema, Ghana, Brasil } \\
\text { (Cocoa) } \\
2011 \text { The Mosaic Company, EE. UU. } \\
\text { (fertilizantes). } \\
2011 \text { Seara Alimentos, Brasil } \\
\text { (productor avícola y porcino) } \\
\text { 2011 Provimi, Argentina (comida } \\
\text { para animals) }\end{array}$ & $\begin{array}{l}\text {-1988: Planta de citricos, Brasil } \\
\text { _1992Fábrica da Comove (procesamiento soja } \\
\text {-1993 Cooper Brasil (citrus) } \\
\text { _2001 Cresciumal, Brasil (planta azucarera- } \\
\text { energética) } \\
\text { _2011 Macrofértil Brasil (fertilizantes) } \\
\text { _2011 Maeda Group (algodón). Se convirtió en el } \\
\text { mayor procesador de algodón. } \\
\text { _2011 Macrofértil (fertilizantes) }\end{array}$ \\
\hline 总 & $\begin{array}{l}\text { 2003 DuPont (semillas } \\
\text { transgénica) } \\
\text { - Fosbrasil S.A.Brasol (fertilizante) } \\
\text { _Solae Brasil (soja) } \\
\text { - AGRI Industries EE.UU. (Soja) } \\
\text {-2006 Southwest lowa Renewable } \\
\text { Ennergy (etanol a partir de maíz). } \\
2007 \text { Ecofuel Argentina (biodisel) } \\
\text {-2008 A.G.D. (Argentina). Terminal } \\
\overline{6} \text { (soja) } \\
\text {-2010 Woodland, Calif. (Molienda } \\
\text { de Arroz). } \\
\text {-2010 Vale S.A Brasil (fertilizantes) } \\
\text {-2012 ITOCHU, U.S. elevador de } \\
\text { exportaciones en Columbia. }\end{array}$ & $\begin{array}{l}\text { - } 1998 \text { Monsanto. Cargill procesará y } \\
\text { entregará cultivos y semillas } \\
\text { producidos por Monsanto. La acción } \\
\text { se extiende a Argentina y Brasil, } \\
\text { áreas en las que Monsanto no es } \\
\text { competitiva debido a la presencia de } \\
\text { Dupont.. También Monsanto ahora } \\
\text { tiene un acceso importante a los } \\
\text { mercados extranjeros donde puede } \\
\text { vender maíz e insecticida, y soja que } \\
\text { puede tolerar la fumigación con el } \\
\text { herbicida Roundup. } \\
\text {-2004 Moisac (fertilizantes) } \\
\text { 2006 Biodiesel Holding and the } \\
\text { Vanden Avenne }\end{array}$ & $\begin{array}{l}2008 \text { Santelisa, se convirtieron el segundo } \\
\text { procesador mundial de azúcar de caña y energía } \\
\text { renovable } \\
2008 \text { A Maggi (Brasil). Una de las principales } \\
\text { empresas procesadoras de granos }\end{array}$ \\
\hline 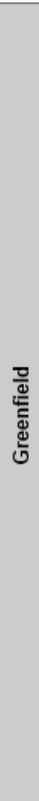 & $\begin{array}{l}\text {-1997 Bunge Pederneiras, Brasil. } \\
\text {-1999 Bunge Fertilizers. Argentina } \\
\text { _1999 Planta de refinación y } \\
\text { trituración de soja más grande de } \\
\text { EE. UU. En Council Bluffs, lowa. } \\
2000 \text { Dos nuevas plantas de } \\
\text { trituración de soja en Sorriso, Mato } \\
\text { Grosso y Uruci, Piauí. Ampliación } \\
\text { de una unidad de producción en } \\
\text { Rondonopolis, Mato Grosso. Planta } \\
\text { de Fatisul en Mato Grosso do Sul } \\
2001 \text { "dos nuevas instalaciones de } \\
\text { trituración noreste y centro-oeste de } \\
\text { Brasil (2001) } \\
\text { 2005 Terminal portuaria del } \\
\text { Complejo Industrial Ramallo, } \\
\text { Argentina (Exportación de granos y } \\
\text { productos derivados) } \\
2007 \text { Ramallo, Argentina } \\
\text { (Fertilizantes) } \\
\text {-Santa Juliana, Brasil. Planta de } \\
\text { azúcar. } \\
\text { 2008 Pedro Afonso, Brasil. Usina } \\
\text { Caña de azúcar } \\
2008 \text { Argentina Planta de Fosfato } \\
\text { (fertilizante) } \\
\text { 2009 Terminal de exportación en } \\
\text { el noroeste de EE.UU. } \\
\text { 2009 Ramallo expansión (crushing } \\
\text { para harina y aceite de soja) } \\
\text { 2010 Cinco nuevos ingenios de } \\
\text { caña de azúcar a las tres plantas } \\
\text { existentes en Brasil }\end{array}$ & $\begin{array}{l}\text {-96 Puerto San Martin, Argentina } \\
\text {-98 Cargill Barreiras, Bahia, Brazil } \\
\text { planta de almacenamiento de soja y } \\
\text { elevadores de última tecnología en } \\
\text { Bahía } \\
\text {-98 Brasil. Duplica la capacidad de } \\
\text { producción (soja) } \\
\text { 2003 Cargill completó en Santarém } \\
\text {-Brasil- un puerto con capacidad de } \\
\text { almacenamiento (soja). }\end{array}$ & 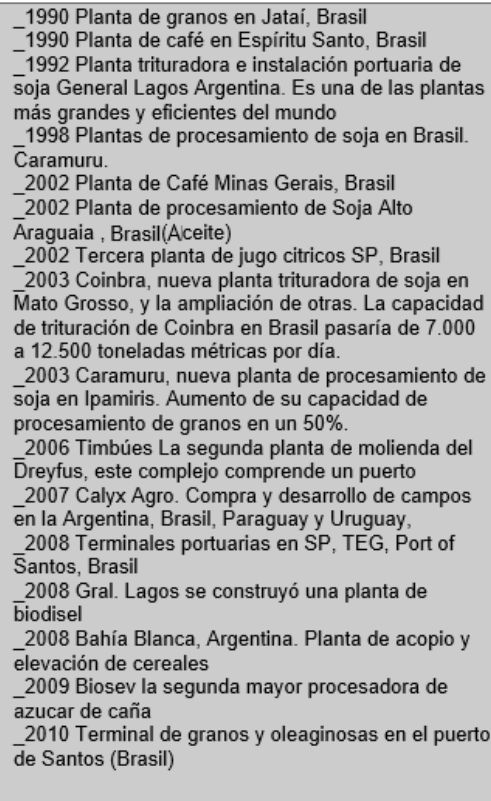 \\
\hline
\end{tabular}

Fuente. Elaboración propia a partir de A) Bunge: Council US Soybean Export, 2011; Goldsmith, Li, Fruin, \& Hirsch, 2004; Soy info center, 2020; https://www.bunge.com/who-we-are/our-history; https://www.bungenorthamerica.com/about/his tory, https://www.bunge-deutschland.de/en/company/history/, https://www.bungeargentina.com/nosotros/pasado-y-pr esente. B) Cargill: Cargill, 2011; Soy info center, 2020; https://www.cargill.be/en/about-cargill. C) Dreyfus: Dreyfus, 2012; Murphy et al., 2012; Soy info center, 2020; https://www.ldc.com/ar/es/; https://www.ldc.com/br/en/who-we-are/ldc-in-brazil/ 
Específicamente en el mercado agropecuario esta dinámica condujo a que BCD frente a un escenario más competitivo y de pérdida de rentabilidad inicien un proceso de integración vertical para la fabricación de productos más elaborados, entre los que se destacan harina y aceite de soja. En relación a la internacionalización de la producción, dicho movimiento expansivo incluyó principalmente a países no desarrollados, tales como Argentina y Brasil. De esta forma, las empresas iniciaron un movimiento hacia la especialización y concentración de la producción y el comercio de productos agropecuarios elaborados de soja.

Si tenemos en cuenta las mayores empresas del rubro en el procesamiento de granos de soja (crushing), ADM y Cargill ya en 1978 concentraban el 15\% cada una (Baines, 2014). Cuestión que implicó un movimiento de inversiones previas considerables en el rubro. Mientras que, según la misma fuente, en el año 1988 entre ADM, Cargill y Bunge contaban con el 71\% de participación en el mercado.

La forma predominante que encontraron estas corporaciones de obtener ganancias es produciendo y comercializando a gran escala y en grandes volúmenes por los bajos márgenes de beneficio que obtienen (Green \& Hervé, 2006), por lo que necesitaron aumentar constantemente las inversiones y desplazar a la competencia en los países de mayor elaboración, como es el caso de Argentina, Brasil y EE.UU. (tabla 1). Esto produjo a nivel mundial una competencia marcada por el manejo de estos elementos, y por el desarrollo tecnológico y en infraestructura desplegada para el transporte terrestre y marítimo. Como respuesta a estos problemas, Bunge y Cargill desde 1980 han reestructurado su producción y comercio orientados a una mayor integración vertical downstream y upstream del mercado agroindustrial (Cuadro 1). Dreyfus va a ingresar más tarde a este mercado, con un fuerte direccionamiento hacia las inversiones greenfield -son las inversiones orientadas a abrir nuevas plantas-. Como observamos en el mismo cuadro, sus adquisiciones, joint venture y greenfield en Argentina, Brasil y EE.UU., además de concentrarse en la producción de harina y aceites de soja también estuvieron dirigidas a la elaboración de fertilizantes y agroquímicos. Otro aspecto a destacar de la integración vertical fueron las joint venture entre Cargill y Bunge con empresas productoras de semillas genéticamente modificadas, Monsanto y Du Pont respectivamente. La asociación fue un medio de fusión empresarial que ofreció casi todas las ventajas de las fusiones pero sin los impedimentos de la ley antimonopolio en varios países (Baines, 2014).

La capacidad que tuvieron estas empresas de adquirir corporaciones o de realizar inversiones greenfield se debe a su experiencia histórica de conocer y haber producido en varios mercados del mundo (Saravia-Matus et al., 2018).

Es de destacar las inversiones para la formación de complejos productivos, de almacenamiento y transporte que realizaron BCD, tales como Council Bluffs, General Lagos, Ramallo, Sorriso, Campinas, entre otros.

Otro soporte fundamental de BCD es el sistema financiero, según el cual el conocimiento que las mismas tuvieron de toda la cadena de valor y la concentración de mercado, le permitió el acceso a información privilegiada y poder establecer estrategias rentables (Salerno, 2017). A su vez, las BCD recurrieron a mecanismos de financiamiento interno para evitar contraer deudas en países en riesgo con altas tasas de interés (Anseeuw et al., 2017). En el caso de Bunge, la empresa mencionó que los diferentes mecanismos financieros con los que contaron les sirvieron como resguardo de fluctuaciones del tipo de cambio, limitándose solo a la función de protegerse frente a la exposición comercial y financiera (Bunge, 2005).

Por otra parte, BCD centraron sus actividades en sus negocios principales (Prahalad \& Hamel, 2006), esto es principalmente en sus productos más rentables como la agroindustria sojera, y por lo tanto iniciando procesos de desinversión en aquellas áreas menos productivas y más distantes del negocio tradicional. 
En definitiva, la integración vertical practicada por estas empresas se basó en consolidar su competitive position (Porter, 1991) al asegurarse ventajas en el poder de negociación de los compradores, en el poder de negociación de los proveedores y neutralizar la rivalidad de los competidores existentes. BCD disputaron el mercado mundial adquiriendo empresas internacionales y locales, con alianzas estratégicas, abriendo nuevas plantas, y modernizaron su infraestructura en los tres países de mayor producción. De esta manera las corporaciones líderes desplazaron a importantes competidores comprando sus empresas, como son los casos de las adquisiciones de CEVAL, Central Soya, La Plata Cereal S.A, y Continental -tabla 1-. A su vez aprovecharon sus conocimientos históricos en el transporte y comercio mundial. Por lo que la integración vertical fue la única forma que tuvieron BCD de avanzar en un creciente contexto competitivo mundial.

El control de la cadena agroindustrial mediante la integración vertical por parte de BCD supuso una mayor dependencia de los productores agropecuarios respecto a las mismas. Los segundos quedaron condicionados a adaptar su actividad a los productos ofrecidos por las transnacionales -tales como las semillas transgénicas y los fertilizantes-, y a una situación de oligopsonio. Mientras que el resto de los productores agroindustriales de harina y aceite de soja en muchos casos no soportaron la competencia y debieron vender sus plantas o cerrar sus negocios.

Las importantes inversiones realizadas durante décadas por BCD en estos países evidencian un caso particular que vincula un país de los llamados desarrollados respecto a dos países en desarrollo. Esta situación pone en duda los marcos teóricos donde se considera como principal motor de las inversiones en los países "subdesarrollados" la occidentalización de sus mercados (Meyer et al., 2009; Peng et al., 2008; White III, Hemphil, Joplin \& Marsh, 2014).

Por otra parte, si bien es cierto que la creciente demanda de estos productos por parte de China motivó a profundizar año a año el nivel tecnológico-productivo de estas industrias (Pierri, 2009), es de destacar que, desde los años ochenta ya se venían realizando importantes inversiones dirigidas a abastecer el mercado interno de los países productores -con excepción de Argentina-, como así también las exportaciones hacia Europa, India y a países del sudeste asiático (Council US Soybean Export, 2011). Este proceso encontró fuertes límites ya a mitad de los años noventa por el aumento de la producción de aceite y harina en China (Council US Soybean Export, 2011). Esto condujo a una especialización al interior de la cadena productiva de soja, según la cual, las corporaciones en EE.UU. y Brasil se dedicaron más a proveer de granos de soja a su mercado interno y a China; mientras que en Argentina expandieron sus exportaciones de harina y aceites (figura 1 y 2 ).

En términos particulares, Bunge en el año 2011 fue el mayor productor internacional de aceite de soja, y sus ventas totalizaron un total de 56,1 miles de millones de U\$S (Bunge, 2011). La empresa en ese año fue el mayor procesador de soja en los EE. UU., operando en trece instalaciones de procesamiento (Council US Soybean Export, 2011). A mitad de los años ochenta la corporación reestructuró su producción siendo su principal negocio la elaboración y comercialización de oleaginosas, y des-invirtiendo en un conjunto importante de productos alimentarios en Argentina, Brasil, Australia y Venezuela (Green \& Hervé, 2006). En la región Latinoamericana su reestructuración fue intensa tras la fundación en 1995 de Bunge Agribusiness Limited. En el año 1997 adquirió un conjunto importante de empresas como I.A.P., Brazilian Fertilizer Manufacturer, y CEVAL la mayor procesadora de soja de Brasil y una de las empresas líderes en Argentina (Tabla 1). Con esto Bunge en Brasil tuvo el segundo mayor polo productivo internacional de productos derivados de soja. En este país también se reestructuraron las actividades de la empresa mediante un proceso de cambio estratégico (Serra et al., 2010), mediante el cual la empresa des-invertió en áreas poco rentables y en general no vinculadas al agronegocio, e invirtió en su principal negocio expandiéndose en la cadena de valor ligada a los granos y fertilizantes (Serra et al., 2010:335). Estas características, como se describirán más adelante, son similares a las estrategias de Bunge en Argentina.

Cargill es la mayor de las comercializadoras de la época, exportando más de 119 mil millones de U\$S en el año 2011 y es la empresa del rubro más grande de EE.UU. luego de su adquisición de Continental 
(Cargill, 2011). En ese año tuvo subsidiarias en América del Norte, América del Sur, en 20 países de Europa, 14 países de Asia y 7 países de África. Cargill también desarrolló una estrategia de integración vertical concentrándose en su negocio principal de productos agropecuarios elaborados, y en especial subproductos de soja. La centralidad de estas actividades se evidencia en el año 2011, en la que la EMN obtuvo beneficios récord principalmente por el desempeño de su unidad de compra en origen y procesado (de cereales y oleaginosas) -(Murphy et al., 2012)-. De por si es conocida su joint venture con Monsanto, el principal productor de semillas transgénicas. Cargill implementó en Brasil un conjunto de inversiones a lo largo de la cadena productiva de aceites y harina de soja tales como la planta de almacenamiento de $800.000 \mathrm{t}$. en Amazonas, y elevadores de última tecnología en Bahía; que le permitió tener una alta performance en infraestructura y logística (tabla 1).

Por último, Dreyfus es una empresa cuyas sedes se sitúan en Francia, China, Argentina, Brasil y Estados Unidos. En el año 2012 trabajaron en la empresa 10.000 empleados; y teniendo en cuenta sus ganancias, ocupa el tercer lugar de las empresas analizadas con un total de 57,1 miles de millones U\$S (LDC, 2012). La estrategia de Dreyfus sigue las mismas tendencias que sus dos principales competidoras, basadas en fortalecer su negocio principal en productos agropecuarios, alimentos agroindustriales y biodiesel, y, aguas abajo, en transporte marítimo, inmuebles, telecomunicaciones, entre otras. Pero fue la empresa que más tarde entró al rubro, y contó con muchas menos actividades en EE.UU. respecto a sus dos máximos competidores. En las últimas décadas también basaron sus estrategias en reestructuraciones en base a la integración vertical aguas arriba y aguas abajo (Wesz Jr, 2016:294).

\section{BCD en Argentina: REESTRUCTURACión PRODUCtiva E INTEgración Vertical 1980-2011}

El desarrollo de la actividad agroindustrial de soja en Argentina respondió a fenómenos internacionales productivos y comerciales que la afectaron, y al modo en que las transnacionales del rubro reestructuraron su producción mediante estrategias de integración vertical.

Por un lado, desde los años setentas según el fenómeno observado en la internacionalización de la producción, la economía latinoamericana se vio afectada por la destacada localización de la actividad industrial de mayor valor agregado en los países desarrollados y en los NICS de Asia (Gereffi, 1990; Gordon, 1988). Esto implicó una pérdida de competitividad fabril en la primer región mencionada, con excepción de los commodities industriales (Katz \& Stumpo, 2001b; Gorenstein \& Ortiz, 2016; Mortimore \& Peres Núñez, 2001).

Frente a estos cambios, durante los años ochenta en Argentina se consolidó un proceso de reestructuración productiva en el cual los productos agroindustriales de soja se convirtieron en uno de los principales exportados. Dicha performance se dio en un contexto de magros resultados de los principales indicadores económicos (Kosacoff,1993). Situación que derivó en un claro entorno favorable para las inversiones en este rubro; y a su vez respecto al país, la actividad se consolidó como una fuente de divisas en un escenario de escasez de las mismas. 
TABLA 2

Participación de productos de soja y primarios en las exportaciones. Argentina 1974-2011 (en \%)

\begin{tabular}{|c|c|c|c|c|c|c|c|c|c|}
\hline & 1974 & 1980 & 1987 & 1991 & 1993 & 1998 & 2002 & 2005 & 2011 \\
\hline $\begin{array}{l}\text { Tortas y harinas de } \\
\text { semillas oleaginosas }\end{array}$ & 1,9 & 3,7 & 13,1 & 10,2 & 10,7 & 7,3 & 10,4 & 9,7 & 12,1 \\
\hline Aceite de soja & & & 3,6 & 4,3 & 4,6 & 5,6 & 5,3 & 5,6 & 6,2 \\
\hline Granos de Soja & & 7,5 & 4 & & 4 & 2 & 4 & 5,7 & 6,5 \\
\hline Total primarios & 38,7 & 33 & 14 & 11 & 17,8 & 18 & 23 & 18,5 & 19,4 \\
\hline Total MOA & 15,9 & 17,5 & 36,2 & 29,2 & 25 & 19,8 & 18,3 & 18,2 & 18,3 \\
\hline Complejo soja & $\underline{1,9}$ & $\underline{11,2}$ & $\underline{\mathbf{2 0}, 9}$ & $\underline{22,2}$ & $\underline{19,5}$ & $\underline{15,4}$ & $\underline{20,1}$ & $\underline{21}$ & $\underline{24,8}$ \\
\hline $\begin{array}{l}\text { Todos los productos (en } \\
\text { millones de dólares) }\end{array}$ & 2.961 & 8.019 & 6.360 & 11.975 & 13.114 & 26.406 & 25.596 & 39.964 & 80.666 \\
\hline
\end{tabular}

Fuente: elaboración propia según CEPAL

La reestructuración productiva agroindustrial se puede observar según el quiebre del predominio agroexportador predominante en la década del setenta (Tabla 2). Si tenemos en cuenta la participación en las exportaciones totales de los primeros 10 productos, entre 1974-1987 los bienes primarios pasaron del 38,7\% al 14,4\%, mientras que el complejo soja -que incluye granos, aceites y tortas y harinas de semillas oleaginosaspasó del 1,9\% al 20,9\%. Además, en estos mismos años las manufacturas de origen agropecuario (MOA) tuvieron altos valores, alcanzando un 36,2\% en 1987, promovidos principalmente por la agroindustria sojera. Este proceso se profundizó a lo largo de la fase 1980-2011 donde el complejo soja pasó del 11,2\% al 24,8\%, y las MOA del 17,5\% al 18,3\%. A esta descripción se le puede sumar el análisis realizado en el apartado 1 en el cual se observó el liderazgo de la producción argentina en aceites de soja y de harinas a nivel mundial.

La importancia que adquirió el sector tiene un fundamento macroeconómico establecido ya en los años ochenta y que perduró en estos años posteriores, convirtiéndose en uno de los principales proveedores de divisas en un contexto de recurrentes déficits. A su vez, la agroindustria sojera en las próximas décadas va a ser uno de los pocos sectores que no estén condicionados por las recurrentes oscilaciones de la economía argentina (incluso en años de grandes crisis como el 2001 y 2002), adquiriendo cierta autonomía respecto a los ciclos económicos locales. El caso incluso cobra mayor importancia si se lo compara con la evolución de la mayoría de los sectores industriales en Argentina (Naspleda, 2020). Este desempeño va a continuar en décadas posteriores -tabla 2-, convirtiéndose el sector en uno de los principales pilares del modo de acumulación argentino, tanto en la época neoliberal como posneoliberal.

Esta cuestión no elimina los importantes problemas que arroja la dependencia del desarrollo del país respecto a la tendencia al "monocultivo" de soja y al desplazamiento de otros cultivos (Dougnac, 2004; Giarracca \& Teubal, 2008), tanto por los daños ecológicos (Giarracca \& Teubal, 2008), como por fomentar un modo de desarrollo que de manera unilateral profundiza la especialización productiva en base a las ventajas comparativas estáticas (Giarracca \& Teubal, 2008; Vidosa, 2020), y basado en la explotación del trabajo rural (Villulla, 2015). 
TABLA 3

Participación de las 5 principales empresas exportadoras de aceite de soja según el volumen de producción, en porcentaje y cantidades

\begin{tabular}{|l|c|c|c|c|c|c|}
\hline \multicolumn{1}{|c}{ Empresas } & 1988 & 1994 & \multicolumn{1}{c}{1997} & 2000 & 2005 & 2010 \\
\hline Cargill & 17 & 18 & 19 & 19 & 20 & 25 \\
\hline Bunge & & & & 9 & 21 & 15 \\
\hline A.G.D. & & 12 & 12 & 13 & 15 & 12 \\
\hline Dreyfus & & & 9 & 15 & 13 & 11 \\
\hline Vicentin & & 9 & 12 & 12 & 13 & 7 \\
\hline Molinos R.P. & & & & & & 9 \\
\hline Indo & 6 & & & & & \\
\hline NIDERA & 6 & 8 & & & & \\
\hline Faca & 5 & & & & & \\
\hline Chabas & 5 & & & & & \\
\hline L. P. Cereal & & 10 & & & & \\
\hline Guipeva & & & 9 & & & \\
\hline Total & 100 & 100 & 100 & 100 & 100 & 100 \\
\hline Total Volumen & 896.733 & 1.449 .206 & 1.931 .741 & $3.142,398$ & 4.924 .680 & 6.027 .824 \\
\hline
\end{tabular}

Fuente: elaboración propia a partir de CIARA, Casanova (2001) y Pierri (2009)

TABLA 4

Participación de las principales 5 empresas exportadoras de harina de soja según el volumen de producción, en porcentaje y cantidades (total)

\begin{tabular}{|c|c|c|c|c|c|}
\hline Empresas & 1988 & 1995 & 2000 & 2007 & 2010 \\
\hline Cargill & 13 & 20 & 16 & 22 & 19 \\
\hline Bunge & & & 11 & 15 & 15 \\
\hline A.G.D. & & 15 & 16 & 14 & 14 \\
\hline Dreyfus & & 8 & 15 & 13 & 11 \\
\hline Vicentin & & 7 & 11 & 12 & 10 \\
\hline Molinos R.P. & & & & 12 & 10 \\
\hline Indo & 7 & & & & \\
\hline I.M.S.A. & 6 & & & & \\
\hline Santa Clara & 5 & & & & \\
\hline Chabas & 7 & & & & \\
\hline L.P. Cereal & & 7 & & & \\
\hline Total & 100 & 100 & 100 & 100 & 100 \\
\hline Total volumen & 4.881 .325 & 6.887 .996 & 12.930 .671 & 27.580 .867 & 28.286 .641 \\
\hline
\end{tabular}

Fuente: elaboración propia a partir de CIARA, Casanova, 2001; y Pierri, 2009

Las tablas 3 y 4 nos permiten identificar la trayectoria de las empresas más importantes que participan en ambos rubros de la producción de derivados de soja. En primer lugar, se observa que el liderazgo de Cargill en todos los años es claro, y su expansión se corresponde con su mayor participación en el mercado exportador de aceite y harina de soja entre los años 1988-2010, si bien en el último su tendencia es más débil. También cabe destacar que Cargill es el máximo exportador de granos de soja.

El segundo lugar en el último año lo ocupa Bunge, y su exportación de aceites muestra una tendencia de mayor concentración en los primeros años del posneoliberalismo (que pasa del 9\% al 25\% entre 2000-2005), y luego cae en el año 2010 un 6\%. Mientras que Bunge en la exportación de harina de soja muestra una 
primera etapa de crecimiento de su concentración que pasa del 11\% al 15\% entre los años 2000-2005, y una segunda etapa de estabilidad en la que mantiene el $15 \%$.

El tercer lugar en importancia lo ocupa la empresa AGD, que desde el año 1994 presenta una tendencia estable en su participación en las exportaciones de aceite de soja -entre los años 1994-2010 es de un 12\%-; y también presenta una participación estable en la concentración de las exportaciones de harina de soja, culminando su desempeño en el año 2010 con un 14\%. En cuarto lugar, Dreyfus también concentra el mercado exportador de aceite y harina de soja desde los años noventa, y en el último año del ciclo analizado presenta una participación del $11 \%$. Cargill, Bunge, Dreyfus, Vicentin y AGD en total representan el 70\% del total exportado en Argentina, y el 69\% de las harinas de soja, lo cual revela en sí mismo su destacada importancia. Por último, un conjunto de empresas de importante participación en el año 1988 va a desaparecer del ranking de las 5 mayores, son los casos de Indo, Santa Clara, I.M.S.A. y Chabás, que en su mayoría serán adquiridas. Indo será adquirida por L. P. Cereal a principios de los años noventa, quien más tarde será propiedad de Bunge al igual que Guipeba y Tankay. Chabas será comprada por A.G.D., e I.M.S.A por Nidera.

El liderazgo de $\mathrm{BCD}$ se debe a sus estrategias aplicadas en el país, que siguieron las tendencias mundiales del desarrollo del sector a nivel global, y responden al tipo de inversiones para la integración vertical llevadas a cabo.

TABLA 5

Plantas de molienda de soja de las EMN en Argentina, ubicación y capacidad de crushing (por día en toneladas), años seleccionados

\begin{tabular}{|c|c|c|c|c|c|c|c|c|c|}
\hline & Localidad & 1989 & 1995 & 1997 & 1998 & 2001 & 2005 & 2006 & 2011 \\
\hline Bunge & Ramallo, Buenos Aires & & & & & & & & 3.000 \\
\hline Bunge & San Jerónimo, Sta. Fe & & & & 1.500 & 1.500 & 2.200 & 2.200 & 2.200 \\
\hline Bunge & Pto. San Martín, Sta. Fe & & & & & 6,400 & 8.000 & 8.000 & 8.000 \\
\hline Bunge & Tancacha, Córdoba (3) & & & & 2.965 & 3.250 & 3.700 & 3.700 & 1.000 \\
\hline Bunge & Pto. San Martín, Sta, Fe 2 & & & & 2.400 & 2.400 & 9.000 & 9.500 & 10.000 \\
\hline Total Bunge & & & & & 6.865 & 13.550 & 22.900 & 23.400 & 21.200 \\
\hline Cargill & Ing. White, Buenos Aires & & & 1.900 & 1.900 & 1.900 & 2,100 & 2.100 & 2.200 \\
\hline Cargill & Pto. Quequén, Buenos Aires & & & 1.500 & 1.500 & 1,700 & 1,700 & 2.000 & 2.000 \\
\hline Cargill & Quebracho, Entre Ríos & 3.100 & 4.800 & 6.900 & 6.900 & 7,500 & 9,000 & 9.000 & 9.000 \\
\hline Cargill & 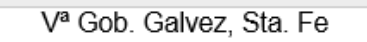 & & & & & & & 6.500 & 13.000 \\
\hline Total Cargill & & & & & 10.300 & 11.100 & 12.800 & 19.600 & 26.200 \\
\hline Dreyfus & Gral. Lagos, Sta. Fe & & 2.900 & 12.000 & 12.000 & 12.000 & 12.000 & 12.000 & 12.000 \\
\hline Dreyfus & Timbues, Sta. Fe & & & & & & & 8,000 & 8.000 \\
\hline Total Dreyfus & & & & & 12.000 & 12.000 & 12,000 & 20.000 & 20.000 \\
\hline Total 3 empresas & & 3.100 & 7.700 & 22.300 & 29.165 & 36.650 & 47.700 & 63.000 & 70.400 \\
\hline$\%$ total & & & & $24 \%$ & $32 \%$ & $40 \%$ & $36 \%$ & $42 \%$ & $40 \%$ \\
\hline Total Industria & & & & 93.345 & 92.530 & 92.548 & 132.068 & 149.318 & 174.206 \\
\hline
\end{tabular}

Fuente: elaboración propia en base a CIARA

Siguiendo la tabla 5 se puede observar que Cargill desde 1989 hasta 2011 fue ampliando la capacidad de crushing, siendo la empresa que más lo incrementó al año 2011 (de 26.200 t por día). Esta corporación fue la primer EMN que realizó importantes inversiones en el país, y la líder mundial. Por otra parte, el grueso de las inversiones en plantas se realizó durante el neoliberalismo, aunque en 2005 ampliaron la capacidad de crushing un 30\% respecto a 2001, y entre 2006-2011 un 12\%. Además, Cargill y Dreyfus abrieron plantas nuevas en 2006. Bunge entró al mercado en 1998, esto refiere a un cambio estratégico de la corporación en la que reforzó su negocio principal a través de su adquisición de Guipeva (Ceval) en 1997, y de La Plata Cereal en 2001. Bunge a su vez desinvirtió en Molinos (1998), Grafa, Centenera y Alba. Dreyfus entró al mercado en 1995 con inversiones en planta en General Lagos y Timbúes. 
TABLA 6

Puertos de las EMN en Argentina, capacidad de almacenaje para aceite (1999)

\begin{tabular}{c|c|c|}
$\begin{array}{c}\text { EMPRESA } \\
\text { TITULAR }\end{array}$ & $\begin{array}{c}\text { Almacenaje } \\
(\mathrm{t})\end{array}$ & $\begin{array}{c}\text { UBICACIÖN } \\
\text { GEOGRÁFICA }\end{array}$ \\
\hline & aceite & \\
\hline Dreyfus & 91.000 & Gral. Lagos \\
\hline Cargill & 30.000 & Pto. San Martín \\
\hline Bunge & 13.650 & $\begin{array}{c}\text { San Gerónimo } \\
\text { Sur, Tancacha }\end{array}$ \\
\hline Total & $\mathbf{1 3 4 . 6 5 0}$ & \\
\cline { 2 - 3 }
\end{tabular}

Fuente: elaboración propia a partir de Casanova, 2001

TABLA 7

Puertos de las EMN en Argentina, capacidad de almacenaje para aceite y harina de soja (2012)

\begin{tabular}{|c|c|c|c|c|}
\hline \multirow[t]{2}{*}{ EMPRESA } & \multicolumn{3}{|c|}{ ALMACENAJE } & UBICACIÖN GEOGRȦFICA \\
\hline & Granos (t.) & Harinas Prot. (t.) & Aceite $(t)$ & \\
\hline Dreyfus & 135.000 & s/datos & 24.000 & km. 462, Timbúes, Sta. Fé \\
\hline Dreyfus & 500.000 & 500.000 & 90.950 & Km 402, Gral. Lagos Arroyo Sec, Sta. Fe \\
\hline Dreyfus total & 635.000 & 500.000 & 114.950 & \\
\hline Cargill S.A. & 78.000 & 18.500 & 34.000 & entre I.White y Galvan, Buenos Aires \\
\hline Cargill S.A. & 415.000 & 285.000 & 82.000 & Km. 454 Pto. San Martin, Sta. Fe \\
\hline Cargill S.A. & 200.000 & no opera & 35.000 & Km. 407,5 \\
\hline Cargill S.A. & & & 18.000 & \\
\hline Cargill total & 693.000 & 303.500 & 169.000 & \\
\hline Bunge S.A. (4) & 500.000 & incluido & & Km. 448 Pto. San Martin, Sta. Fe \\
\hline Terminal 6 S.A. & 115.000 & 600,000 & & Km. 456 Pto. San Martin, Sta. Fe \\
\hline Terminal 6 S.A. & Compartido & Muelle Sur & & Km. 455,5 Pto. San Marti, Sta.Fe \\
\hline Bunge S.A. & 200.000 & a futuro & 20.000 & Ramallo Km. 322, Buenos Aires \\
\hline Bunge total & 815.000 & 600.000 & 20.000 & \\
\hline Total EMN & 2.143 .000 & 1.403 .500 & 303.950 & \\
\hline
\end{tabular}

Fuente: elaboración propia a partir de CIARA

Siguiendo las tablas 5 y 6 se puede destacar que la mayoría de los puertos en el año 2012 se encuentran en la misma zona que las plantas de molienda de soja. Esto constituye una de las ventajas más importantes de la competitividad de las EMN en Argentina respecto a Brasil y EE.UU., porque en esos países la distancia entre las regiones de producción y la zona portuaria pueden estar a más de 1000 millas (López et al., 2010; Meade, Mc. Bride, Puricelli, Valdes, Hoffman, Foreman, \& Dohlman, 2016). A su vez, en el año 2012 se registran 6 nuevos puertos de las EMN, que implica un aumento de la integración vertical. Los mismos amplían la capacidad de almacenaje de aceites en un 136\% respecto a 1999, mostrando una expansión de la integración aguas abajo. La mayoría de los puertos cuentan con capacidad de almacenaje de granos, lo que permite a las empresas concentrar sus actividades también en este rubro.

Las principales exportadoras de aceite y harina de soja a su vez son líderes en las exportaciones de grano de soja. Cargill en el año 2007 fue la principal empresa exportadora de esta mercancía acaparando el 20,4\% de las cantidades exportadas, Bunge es la quinta empresa exportadora con el 12,8\% y Dreyfus es la sexta empresa 
con el 12,8\% (Giancola et al., 2009). El manejo parcial del principal insumo de su producción les permitió sumar una ventaja competitiva.

En definitiva, el crecimiento del sector agroindustrial fue el resultado del control gradual de la cadena de valor de soja por parte de un conjunto de pocas empresas que incluyeron desde los años ochenta a Cargill y a empresas locales que luego fueron adquiridas. Posteriormente, Bunge y Dreyfus en los años noventa y durante el próximo decenio realizaron un conjunto de inversiones decisivas para ser parte de los líderes del mercado, las mismas fueron efectuadas producto de la presión competitiva a nivel mundial y el crecimiento destacado de Cargill. En ese sentido cronológico, las principales empresas que participaron en Argentina en todo el periodo coordinaron un conjunto de estrategias orientadas a la integración vertical de la agroindustria, siendo las BCD las que pudieron llevarlas a cabo de manera exitosa aprovechando su posición competitiva local e internacional. El aumento de la concentración e internacionalización en el rubro por parte de las corporaciones marca el manejo de las mismas de la cadena de valor por las mejores condiciones en su competive position (Porter, 1991), al asegurarse ventajas en el poder de negociación de los compradores, en el poder de negociación de los proveedores y neutralizar la rivalidad de los competidores locales existentes. Las mismas respondieron, como vimos en el apartado anterior, a la necesidad que presenta la competencia en este mercado de un continuo desarrollo productivo, tecnológico y logístico para el control de la cadena agroindustrial, el logro de saltos de productividad acorde a la demanda mundial, y para el desplazamiento de competidores.

Por lo que las transformaciones de la agroindustria no responden en sus inicios a la dinámica neoliberal como sostienen varios autores (Giarracca \& Teubal, 2008; Grondona \& Burgos, 2015; Gutman \& Gorenstein, 2005; Regunaga, 2010; Sly, 2017; Vidosa, 2020). Responde a como se sucedió históricamente la dinámica competitiva mundial a partir de las estrategias de estas empresas, y la configuración de un modelo productivo y comercial con eje en la integración vertical. Si bien es de destacar que durante el neoliberalismo se profundizó la transnacionalización y concentración de su producción por el contexto de apertura económica favorable a las Empresas alimentarias (Gutman \& Gorenstein, 2005), dando como resultado el ingreso de LCD y Bunge.

Bajo este último aspecto, durante los años noventa en Argentina las nuevas condiciones favorables para el desarrollo de este sector y de las corporaciones fueron dadas por el incremento de la competencia coercitiva (O'Connor, 2010). La mismo consistió en tres procesos: la racionalización del estado, la contestabilidad del mercado y la movilidad de factores. La racionalización del estado es una forma de ordenar la economía de un país bajo la preminencia del mercado que busca igualar, mediante diferentes mecanismos, las condiciones de competencia entre las empresas extranjeras y las empresas locales. Las medidas que más se encaminaron en esta dirección fueron el desplazamiento de la inversión pública hacia la privada, la disminución de los aranceles a las importaciones -reduciéndose para el año 1992 el arancel máximo a 20\%, se desagregó el arancel llevándolo a $0 \%$ para el caso de bienes de capital-y la eliminación de restricciones a las operaciones de empresas multinacionales (Bisang y Gómez, 1999).

Las condiciones favorables para las empresas transnacionales en este contexto fueron aprovechadas por Bunge y Dreyfus que se establecieron en el país. Mientras que Cargill en estos años multiplicó 3,6 veces su capacidad de crushing (tabla 5). Como resultado la agroindustria sojera se concentró e internacionalizó, cuestión reflejada en que las cinco empresas que más contribuyen a las exportaciones de harinas entre 1988-2000 pasaron de concentrar el 38\% al 69\% del total, mientras que respecto a las harinas de soja para los mismos años pasaron de concentrar el 39\% al 68\%, liderando el crecimiento en ambos rubros BCD.

Esta situación contradice a los autores que explican la evolución de la agroindustria sojera en Argentina frente a una política antimonopolio débil en el neoliberalismo (Delvenne et al., 2013), o de mayor horizontalidad en el sector por el paso de una producción vertical a una producción en red (Bisang et al., 2008). Por lo que no se puede considerar que las BCD simplemente transformaron las formas tradicionales de producción en un nuevo modelo productivo (Regunaga, 2010). 
El aprovechamiento del manejo de la cadena de valor sojera por parte de las empresas que concentran la producción significó un aumento destacado de la producción de harinas y aceites de soja muy por encima de la exportación del grano, como si sucedió en EE.UU. y Brasil (Council US Soybean Export, 2011). Esto no se condice con quienes sostienen que hubo un fuerte crecimiento de la producción y exportaciones de productos primarios o una reprimarización de la economía (Giarracca \& Teubal, 2008; Palmisano, 2019). Como hemos visto, para la formación de un complejo agroindustrial competitivo a nivel mundial hicieron falta importantes inversiones en diferentes puntos de la cadena de soja, tendientes a un aumento de productividad, junto a la resolución de problemas logísticos en el escenario global. De esta manera, las fronteras entre la producción primaria, industria, y servicios se tornó cada vez más difusa (Anlló et al., 2010), pero en el marco de un proceso de concentración y de integración vertical por parte de un conjunto reducido de empresas.

Otros autores explican el destacado desarrollo del cultivo sojero y el abaratamiento de sus productos durante los años noventa por el bajo costo del paquete tecnológico (tanto de la semilla transgénica como el herbicida) dado por la no aplicación de la patente de Monsanto (Delvenne et al., 2013). Este punto es cuestionable debido a la alianza estratégica entre Cargill y Monsanto para introducir el paquete tecnológico en países donde no era competitivo, como el caso argentino, y para realizar pruebas acerca de la eficacia del producto y de efectos adversos (Soy info center, 2020). En este sentido, no parece un accidente el hecho de que Monsanto en los años noventa no va a obtener derechos de propiedad intelectual por solicitar las patentes correspondientes cuando ya había expirado el plazo legal para hacerlo (Pérez Trento, 2019). Tampoco es menor el hecho de que EE.UU. y Argentina se convertirán en los principales países que van a utilizar las semillas transgénicas, y ya en el año 2000, a tres años de su disponibilidad en el mercado argentino, casi el 90\% del área sembrada involucró soja genéticamente modificada (Regunaga, 2010). Por lo que mediante su instalación en el país Monsanto y Du Pont ganaron una considerable cuota de mercado en pocos años. A su vez, la adopción del paquete tecnológico de siembra directa, semilla transgénica y herbicida requiere un mayor uso de fertilizantes (Bernado, 2020), actividad que, como hemos visto, es dominada por BCD.

\section{FIGURA 3}

Producción y valor de las exportaciones (eje derecho) de aceite y harina de soja en Argentina, 1988-2011

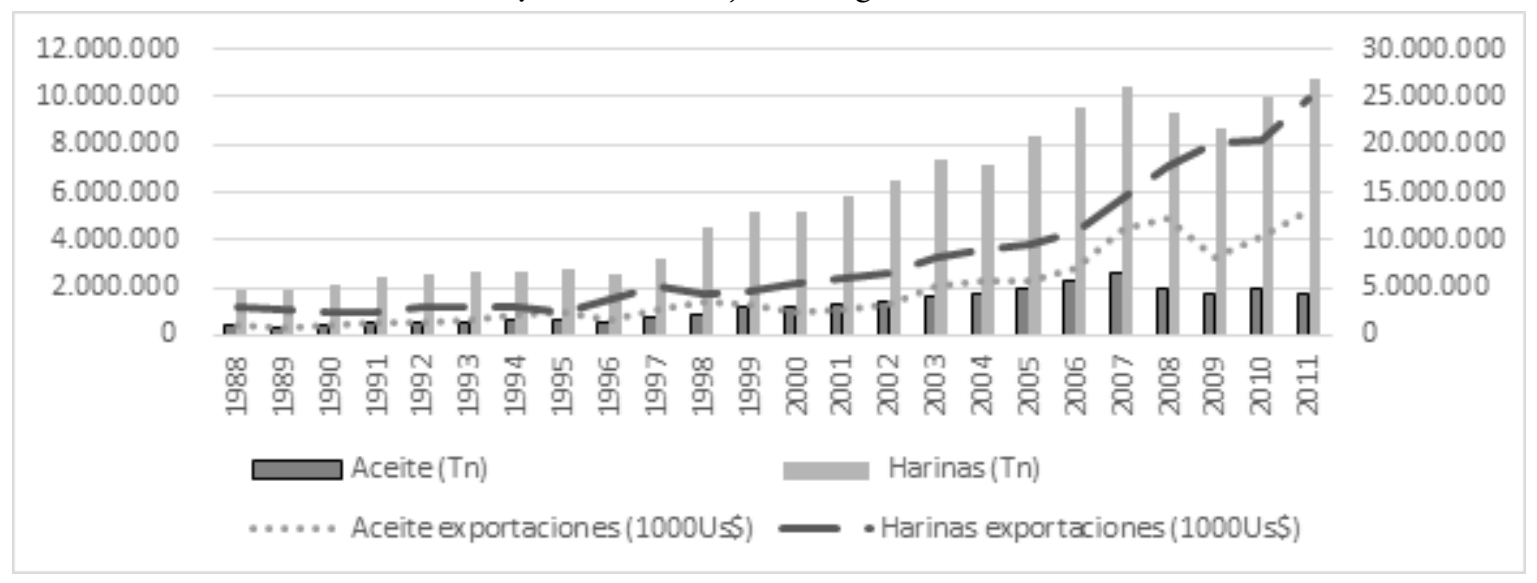

Fuente: elaboración propia según FAO

Por otra parte, la producción y los valores exportados de aceite y harina de soja mostró una tendencia al crecimiento incluso en los años de crisis de convertibilidad (1998-2002), y una inclinación al incremento aún más acentuado en los años de posconvertibilidad (2002-2011) - figura 3-. Además, estas producciones entre los años 1988-2011 presentaron una considerable tendencia al aumento de su producción y exportaciones, que es más significativa si los comparamos con la evolución industrial inestable de la época (Naspleda, 2020). 
En los años de crisis del neoliberalismo y posneoliberalismo estos subsectores tuvieron un crecimiento histórico, que se corresponde con el aumento de las inversiones en infraestructura (Tabla 5 y 7 ). Los beneficios que estos sectores lograron por la devaluación del peso en 2002 (Varesi, 2020) no fueron sustancialmente afectados por el nuevo esquema de derechos a las exportaciones adoptado por el estado argentino en 2002, 2007 y 2008. El mismo en el último año mencionado pasó a ser para granos del 35\%, y para aceites y harinas del $32 \%$.

TABLA 8

Exportaciones totales de BCD en Argentina y Brasil en millones de U\$S

\begin{tabular}{|c|c|c|c|c|c|c|c|c|c|c|c|c|c|c|}
\hline & \multicolumn{2}{|l|}{2005} & \multicolumn{2}{|l|}{2006} & \multicolumn{2}{|l|}{2007} & \multicolumn{2}{|l|}{2008} & \multicolumn{2}{|l|}{2009} & \multicolumn{2}{|l|}{2010} & \multicolumn{2}{|l|}{2011} \\
\hline & Argentina & Brasil & Argentina & Brasil & Argentina & Brasil & Argentina & Brasil & Argentina & Brasil & Argentina & Brasil & Argentina & Brasil \\
\hline Bunge & 2.400 & 2.100 & 2.450 & 2.050 & 1.900 & 3.000 & 3.750 & 5.000 & 2.300 & 4.050 & 3.350 & 4.050 & 3.950 & 6.050 \\
\hline Corgill & 3.400 & 1.100 & 3.600 & 1.100 & 4.350 & 1.900 & 5.650 & 2.000 & 4.000 & 2.000 & 4.400 & 3.000 & 5.800 & 4.000 \\
\hline Dreyfus & 1.000 & 500 & 1.200 & 700 & 1.100 & 1.500 & 3.150 & 1.850 & 3.400 & 1.600 & 2.950 & 1.850 & 2.600 & 2.500 \\
\hline
\end{tabular}

Por último, se puede observar que en la posconvertibilidad el aumento en la producción y en las exportaciones de aceite y harina de soja se corresponde con el incremento de los valores exportados por las BCD (Tabla 8). Entre los años 2005-2011 Bunge creció un 65\%, Cargill 71\% y Dreyfus 160\%. Si bien en Brasil estas empresas crecieron más, con un $188 \%, 264 \%$ y $400 \%$ respectivamente, lo que muestra una pérdida de competitividad argentina.

\section{Conclusiones}

Como se ha expuesto a lo largo del trabajo, la estrategia de integración vertical por parte de BCD en Argentina, Brasil y EE.UU. fue producto del avance de la internacionalización de la producción desde principios de los años setentas. La misma condujo a una reestructuración de sus inversiones hacia bienes más elaborados por la pérdida de rentabilidad de su actividad comercial y el aumento de la competencia. Para esto, estas corporaciones adquirieron empresas internacionales y locales, iniciaron alianzas estratégicas, abrieron nuevas plantas, y modernizaron su infraestructura. La Integración vertical estuvo dirigida principalmente a sumar a las actividades de producción de aceite y harina una serie de eslabones comerciales y productivos. De manera tal que la integración vertical a nivel global les permitió a BCD consolidar su competive position al asegurarles ventajas en el poder de negociación frente a los compradores y los proveedores, y neutralizar a los competidores locales e internacionales. A su vez, la capacidad que tuvieron estas empresas de adquirir corporaciones o de realizar inversiones greenfield se debe a su experiencia histórica de conocer y haber producido en varios mercados del mundo. Como resultado las BCD aprovecharon las ventajas competitivas que presentaron los tres principales países productores, convirtiéndose de esta forma en los líderes del mercado agroindustrial.

En Argentina este proceso se relacionó a fenómenos internacionales y locales vinculados al impacto de la internacionalización productiva en el país. En primer lugar, por el modo en que aconteció una reestructuración productiva desde los años ochenta en el país, según la cual, los productos agroindustriales de soja se convirtieron en uno de los principales exportados. Esta situación le dio al complejo de soja un carácter estratégico para el país como proveedor de divisas, cuestión que progresará notablemente durante los años neoliberales y posneoliberales. Como vimos en el artículo, este escenario aumentó las expectativas del sector siendo su crecimiento resultado de continuas inversiones. Las mismas condujeron al control gradual de la cadena de valor de soja por parte de un conjunto de pocas empresas, que incluyeron desde los años ochenta a Cargill y a empresas locales, y posteriormente en los años noventa a Bunge y Dreyfus. Las mismas respondieron a la necesidad que presenta la competencia en este mercado de un continuo desarrollo 
productivo, tecnológico y logístico, para el control comercial de la cadena agroindustrial, el logro de saltos de productividad acorde a la demanda mundial, y para el desplazamiento de competidores. En términos de la competencia en Argentina, Cargill desde los inicios del proceso dominó el sector, y esto se relaciona con la temprana puesta en marcha de la integración vertical a nivel mundial y su dominio de la actividad en el escenario internacional. Mientras que Bunge y Dreyfus aplicaron la integración vertical en el país de estudio frente a la amenaza competitiva de Cargill durante el neoliberalismo, aprovechando las ventajas dadas por la competencia coercitiva que les permitió desplazar a parte importante de la competencia local adquiriendo empresas o mediante la apertura de plantas sin restricciones. Posteriormente durante el posneoliberalismo las BCD aprovecharán las ventajas comerciales de la devaluación, y seguirán invirtiendo en el país en mayor medida agrandando sus plantas o abriendo nuevas.

La mayor dependencia que presenta la estructura productiva argentina en la fase de estudio respecto a la evolución agroindustrial es lo distintivo respecto a ambos países por el lugar que tuvo Argentina en la internacionalización productiva. Esta dependencia es la que condiciona el desarrollo del país a la lógica de acumulación de las grandes empresas agroindustriales.

Por último, es preciso profundizar en futuros estudios en el análisis de los años siguientes de los aquí examinados por la existencia de importantes transformaciones que dan lugar a una nueva etapa. Estos cambios refieren a nuevas estrategias de diversificación de actividades de las empresas examinadas; en un contexto que abarca fenómenos tan complejos como el escenario de poscrisis mundial, y un contexto económico inestable en argentina.

\section{Bibliografía}

Anlló, G., Bisang, R., \& Katz, J. (2015). Aprendiendo con el agro argentino (Documento N IDB-DP-379). Recuperado de Banco Interamericano de Desarrollo: https://publications.iadb.org/publications/spanish/document/Apren diendo-con-el-agro-argentino-De-la-ventaja-comparativa-a-la-ventaja-competitiva-El-rol-de-las-KIBs.pdf.

Anlló, G., Bisang, R., \& Salvatierra, G. (2010). Cambios estructurales en las actividades agropecuarias: de lo primario a las cadenas globales de valor. Santiago de Chile: Naciones Unidas.

Anseeuw, W., Roda, J.-M., Ducastel, A., \& Kamaruddin, N. (2017). Global strategies of firms and the financialization of agriculture. En Sustainable Development and Tropical Agri-chains (pp. 321-337). Springer.

Baines, J. (2014). Food price inflation as redistribution: Towards a new analysis of corporate power in the world food system. New Political Economy, 19(1), 79-112.

Bernado, R. G. (2020). Impacto del modelo productivo agrario en la industria química argentina en los años recientes (2006-2017). H-industri@: Revista de historia de la industria, los servicios y las empresas en América Latina, 26, 85-108.

Bisang, R. (2011). ¿Agro y recursos naturales en la Argentina? ¿Enfermedad maldita o desafío a la inteligencia colectiva?. Boletín Informativo Techint, 336, 63-83. Recuperado de https://www.researchgate.net/publication/285021222_Agro_y_Recursos_Naturales_en_la_Argentina_E nfermedad_Maldita_o_Desafio_a_la_Inteligencia_Colectiva

Bisang, R., Anlló, G., \& Campi, M. (2008). Una revolución (no tan) silenciosa. Claves para repensar el agro en Argentina. Desarrollo Económico, 48(190/191), 165-207.

Bisang, R., Anlló, G., Campi, M., \& Albornoz, I. (2009). Cadenas de valor en la agroindustria. En,B. Kosacoff y Rubén Mercado (2009), La Argentina ante la nueva internacionalización de la producción. Crisisy oportunidades, Buenos Aires, CEPAL-ONUD, 218-276.

Bisang, R., \& Gómez, G. (1999). Las inversiones en la industria argentina en la década de los años noventa. Buenos Aires: CEPAL. http://repositorio.cepal.org//handle/11362/7503

Brenner, R. (2006). The economics of global turbulence: The advanced capitalist economies from long boom to long downturn, 1945-2005. Verso. 
Bunge (2005). Annual Report.

Bunge (2011). Annual Report.

Cargill (2011). Annual Report.

Casanova, M. (2001). Comercialización de soja y sus derivados: Concentración de las exportaciones e integración vertical. Departamento de Capacitación y Desarrollo de Mercados. Bolsa de Comercio de Rosario.

Clapp, J. (2014). Financialization, distance and global food politics. The Journal of Peasant Studies, 41(5), 797-814.

Delvenne, P., Vasen, F., \& Vara, A. M. (2013). The "soy-ization" of Argentina: The dynamics of the "globalized" privatization regime in a peripheral context. Technology in society, 35(2), 153-162.

Dougnac, G. M. (2004). Apuntes acerca de la historia de la soja en la Argentina. Elementos para delinear experiencias comparadas. Documentos del CIEA.

EE.UU. Council US Soybean Export. (2011). How the global oilseed and grain trade works. The U.S. Soybean Export Council (USSEC). Recuperado de: https://ussec.org/

Informa Economics (2016). Farm to Market a Soybean's Journey from Field to Consumer. Memphis, agosto. Recuperado de http://www.informaecon.com

Felipe, J., \& Mehta, A. (2016). Deindustrialization? A global perspective. Economics Letters, 149, 148-151.

Garrett, R. D., Rueda, X., \& Lambin, E. F. (2013). Globalization's unexpected impact on soybean production in South America: Linkages between preferences for non-genetically modified crops, eco-certifications, and land use. Environmental Research Letters, 8(4), 044055.

Gereffi, G. (1990). Paths of industrialization: An overview. Manufacturing Miracles: Paths of Industrialization in Latin America and East Asia, 3, 31.

Giancola, S. I., Salvador, M. L., Covacevich, M., Oliveros, I.-E., Iturrioz, G., \& Anguil, I.-E. (2009). Análisis de la cadena de soja en la Argentina. Instituto Nacional de Tecnologia Agropecuaria, Buenos Aires (Argentina) (Proyecto Específico 2742). Recuperado de INTA: https://inta.gob.ar/sites/default/files/script-tmp-cadena_soja.pdf

Giarracca, N. y Teubal, M. (2008). Del desarrollo agroindustrial a la expansión del agronegocio: El caso argentino. En Bernardo Mançano Fernandes, Campesinato e agronegócio na América Latina: a questão agraria atual (pp. 139-164). Sao Pablo: CLACSO Livros.

Goldsmith, P. D., Li, B., Fruin, J. E., \& Hirsch, R. (2004). Global shifts in agro-industrial capital and the case of soybean crushing: Implications for managers and policy makers. International Food and Agribusiness Management Review, 7(1030-2016-82664), 87-115.

Gordon, D. (1988). The global economy: new edifice or crumbling foundations? New Left Review, 168, 24-64.

Gorenstein, S., \& Ortiz, R. (enero, 2016). Empresas transnacionales en la agricultura y la producción de alimentos en América Latina y el Caribe. Documento de Trabajo, Fundación Foro Nueva Sociedad (NUSO), Buenos Aires.

Gras, C. (2012). Los empresarios de la soja: Cambios y continuidades en la fisonomía y composición interna de las empresas agropecuarias. Mundo agrario, 12(24), 00-32.

Green, R., \& Hervé, S. (2006). IP-Traceability and grains traders: ADM, Bunge, Cargill, Dreyfus (Cahier n²006-03). Recuperado de Laboratoire Sur Les Organisations Industrielles Dans l'AgroAlimentaire: https://hal.inrae.fr/h al-02822998/document.

Grondona, V., \& Burgos, M. (2015). Estimación de los precios de transferencia. El caso del complejo sojero (Documento de trabajo N 71). Recuperado de CEFIDAR: http://www.iade.org.ar/noticias/estimacion-de-los-precios-de-transf erencia-el-caso-del-complejo-sojero .

Gutman, G., \& Gorenstein, S. (diciembre, 2005). Las transnacionales alimentarias en Argentina. Dinámica reciente e impactos territoriales. Trabajo presentado en Primeras Jornadas de Economía Regional Comparada-Simposio de Economía de los Agronegocios en el Mercosur, Porto Alegre.

He, R., Zhu, D., Chen, X., Cao, Y., Chen, Y., \& Wang, X. (2019). How the trade barrier changes environmental costs of agricultural production: An implication derived from China's demand for soybean caused by the US-China trade war. Journal of Cleaner Production, 227, 578-588. 
Katz, J., \& Stumpo, G. (2001). Regímenes sectoriales, productividad y competitividad internacional. Revista de la CEPAL, 75, 137-159.

Kosacoff, B. (1993). La industria argentina: Un proceso de reestructuración desarticulada. Buenos Aires: CEPAL. http s://www.cepal.org/es/publicaciones/2747-la-industria-argentina-un-proceso-reestructuracion-desarticulada

LDC (2012). Annual Report.

López, A., Ramos, D., \& Starobinsky, G. (2010). A Study of the Impact of China's Global Expansion on Argentina: Soybean Value Chain Analysis. Universidad Nacional Autónoma de México (UNAM), Facultad de Economía.

Meade, B., McBride, W. D., Puricelli, E., Valdes, C., Hoffman, L., Foreman, L., \& Dohlman, E. (2016). Corn and soybean production costs and export competitiveness in Argentina, Brazil, and the United States. United States Department of Agriculture.

Meyer, K. E., Estrin, S., Bhaumik, S. K., \& Peng, M. W. (2009). Institutions, resources, and entry strategies in emerging economies. Strategic management journal, 30(1), 61-80.

Mortimore, M., \& Peres Núñez, W. (2001). La competitividad empresarial en América Latina y el Caribe. Revista Cepal, 74, 37-59.

Murphy, S., Burch, D., \& Clapp, J. (2012). Cereal secrets: The world's largest grain traders and global agriculture. Recuperado de Oxfam research reports: https://www.oxfam.org/en/research/cereal-secrets-worlds-largest-grai n-traders-and-global-agriculture

Naspleda, F. D. (2020). Contribuciones al examen del crecimiento de la acumulación industrial argentina en la posconvertibilidad (2005-2011) y su comparación con la fase de incremento neoliberal (1993-1998). Revista Galega de Economia, 29(1), 6063.

O'Connor, J. (2010). Marxism and the three movements of neoliberalism. Critical Sociology, 36(5), 691-715.

Oliver, L. G., \& Sánchez, R. G. (2016). Las cuatro grandes empresas comercializadoras y los precios internacionales de los alimentos. Economia Informa, 400, 24-39.

Palmisano, T. (2019). El agronegocio sojero en Argentina: Modelo extractivo en los mundos rurales. Revista Economía, 68(107) (mayo 2016), 13-33.

Peng, M. W., Wang, D. Y., \& Jiang, Y. (2008). An institution-based view of international business strategy: A focus on emerging economies. Journal of international business studies, 39(5), 920-936.

Pérez Trento, N. (2019). Dos décadas de conflicto en torno al uso propio de semillas de soja genéticamente modificada en Argentina: Fases del enfrentamiento, acumulación de capital y actores sociales (1996-2018). Mundo Agrario, 20(43).

Pierri, J. (2008). El desempeño de las grandes empresas y las cooperativas en la "sojización". Los casos de Cargill y de la Asociación de Cooperativas Agrarias (Documentos 3, 69-95). Recuperado de CIEA: https://repositoriosdigitale s.mincyt.gob.ar/vufind/Record/BDUBAFCE_8a487b5cce415c59fd62f8f6afb1c2ec

Pierri, J. (2009). Grandes empresas y cooperativas en el comercio exterior del complejo sojero, 1990-2006 (Documentos 4, 147-163). Recuperado de CIEA: http://bibliotecadigital.econ.uba.ar/econ/collection/docuci ea/document/docuciea_n4_06

Pierri,J., \& Junior, V.J. W. (2017). La sojización en Argentina y Brasil (1980/2014): Influencia de las políticas públicas, de las empresas transnacionales y de la estructura económica dependiente. Extensão Rural, 24(1), 20-41.

Porter, M. E. (1982). Ventaja Competitiva: Creación y sostenimiento de un desempeño superior. REI CECSA, Argentina.

Prahalad, C. K., \& Hamel, G. (2006). The core competence of the corporation. Strategischeunternehmungsplanung, (pp. 275-292). Springer.

Regunaga, M. (2010). Implications of the organization of the commodity production and processing industry: The soybean chain in Argentina. World Bank, LCSSD Food Papers Series.

Salerno, T. (2017). Cargill's corporate growth in times of crises: How agro-commodity traders are increasing profits in the midst of volatility. Agriculture and Human Values, 34(1), 211-222. https://doi.org/10.1007/s10460-01 6-9681-8 
Saravia-Matus, S., Matus, J. S., Sotomayor, O., \& Rodriguez, A. (2018). Investment strategies in the Latin American agri-business sub-sectors of agricultural commodities, biofuels and meat chains. Journal of Agribusiness in Developing and Emerging Economies, 8 (2): 320.

Schnepf, R., Dohlman, E., \& Bolling, C. (2001). Agriculture in Brazil and Argentina: Developments and Prospects for Major Field Crops. Economic Research service. U.S. Department Of Agriculture. Recuperado de: https://www .ers.usda.gov/publications/pub-details/?pubid $=40353$

Serra, F. A. R., Ferreira, M. P., Contrigiane, E., \& Fiates, G. G. S. (2010). Reescrevendo uma trajetória a partir de mudanças estratégicas: Um estudo de caso na Bunge. REGE Revista de Gestão, 17(3), 331-352.

Sly, M. J. H. (2017). The Argentine portion of the soybean commodity chain. Palgrave Communications, 3(1), 1-11.

Soy Info Center (2020). History of early, small and other u.s. soybean crushers (1910-2020). Recuperado de Soy Info Center: https://www.soyinfocenter.com/free-online-books.php

Varesi, G. Á. (2020). Circuito productivo sojero y conflicto agrario en la Argentina. El año 2008 como hito y punto de inflexión. Mundo Agrario, 21 (48), e154.

Vidosa, R. (2020). Acumulación y arreglos institucionales en torno al circuito oleaginosa de la soja en Argentina (2008-2019). En A. Rofman, Circuitos productivos regionales y estrategias de desarrollo en la argentina del siglo xxi. IADE, Buenos Aires ,137-164.

Villulla, J. M. (2015). Las cosechas son ajenas: Historia de los trabajadores rurales detrás del agronegocio. Editorial Cienflores, Buenos Aires.

Wesz Jr, V.J. (2016). Strategies and hybrid dynamics of soy transnational companies in the Southern Cone. The Journal of Peasant Studies, 43(2), 286-312.

White III, G. O., Hemphill, T. A., Joplin, J. R., \& Marsh, L. A. (2014). Wholly owned foreign subsidiary relationbased strategies in volatile environments. International Business Review, 23(1), 303-312. 